\title{
Heat Transfer on MHD Rotating non-Newtonian Fluid Flow through Parallel Plate Porous Channel
}

\author{
B.Lakshmanna ${ }^{1^{*}}$ and S.Venkateswarlu' ${ }^{2}$ \\ ${ }^{1}$ Research Scholar, Dept of Mathematics, Rayalaseema University, Kurnool, Andhra Pradesh 518007, India. \\ ${ }^{2}$ Professor, Dept. of Mathematics, RGM College of Engineering and Technology, Nandyal, Kurnool, AP, India.
}

\begin{abstract}
In this paper, we consider hydromagnetic convective flow of an electrically conducting second order fluid through a rotating porous channel. The governing equations are framed using Brinkman model. The exact solutions of the velocity and the temperature distributions are evaluated analytically using Laplace transform technique and which consist of the both steady and transient states. Thought is centered on the physical character of the solutions, and the construction of the various kinds of boundary layers outward appearance on the plates. The ultimate steady state velocity and temperature distributions are numerically discussed for various values of the flow parameters. The shear stresses and the Nusselt number are tabulated and discussed.
\end{abstract}

Keywords: Convective flows, heat source, heat transfer, porous medium, rotating channels and unsteady state flows

\section{INTRODUCTION}

The flow through porous medium is very important particularly in the fields of agricultural engineering and technology for irrigation processes, especially in petroleum industry to study petroleum extraction process and transport, also in chemical engineering and technology for filtration and purification processes. The MHD flow in a rotating harizontal Porous channel has been studied by Sing and Alphonsa [1]. Krishna and Suneetha [2] discussed the Hall current effects on unsteady flow of Newtonian fluid between two rigid nonconducting rotating plates. Recently, Hall effects on an unsteady MHD flow of a viscous incompressible electrically conducting fluid in a horizontal porous channel with variable pressure gradient in a rotating system have discussed by Sanatan Das and Rabindranath Jana [3]. Few attempts in this direction are made by Narahari [4]. Significant concern has been originated in the study of magnetic field and the electrically conducting fluids flow, while medium is porous [5]. MHD free convection flow of an incompressible viscous fluid near an oscillating plate embedded in a porous medium has been presented by Khan et al. [6]. Therefore, many researchers have studied free convection flow past a vertical plate with thermal radiation [7-9]. Recently, Krishna and
Prakash [10] discussed the hall current effects on Unsteady MHD flow in a Rotating parallel plate channel bounded by Porous bed on the Lower half. Krishna [11] discussed the unsteady flow of an incompressible electrically conducting viscous fluid in a rotating porous media, with a variable pressure gradient and in the presence of hall current. Lakshmanna and Venkateswarlu[12] studied the MHD flow of an incompressible fluid with heat and mass transfer over a vertical plate in the presence of magnetic field with soret and dufour effects, chemical reaction and convective heat exchange at the surface with the surrounding. Recently, Lakshmanna and Venkateswarlu [13,14] discussed the (a)Hall effects on the unsteady free convective two dimensional flow of a viscous incompressible electrically conducting second fluid over an infinite vertical porous plate under the influence of uniform transverse magnetic field with time dependent permeability, oscillatory suction.(b)Unsteady MHD flow through a loosely packed porous medium in an impulsively started vertical plate with variable heat and mass transfer.

In this paper, we have considered the hydromagnetic convective flow of an electrically conducting second order fluid through a rotating porous channel using Brinkman model.

\section{FORMULATION AND SOLUTION OF THE PROBLEM:}

We have considered the unsteady hydromagnetic convective flow of an electrically conducting second order fluid through porous medium two parallel non conducting plates under a uniform transverse magnetic field $H_{o}$. At initial stage, both the plates and the fluid rotate with the same angular velocity $\Omega$. At $t>0$, the fluid obsessed by a invariable pressure gradient parallel to the plate and in addition the lower plate performs non-torsional oscillation in its individual plane. We promoted the plates are cooled or heated by a unvarying temperature gradient in same direction parallel to the plane at the plates. The physical configuration of the problem is as shown in Figure. 1. 


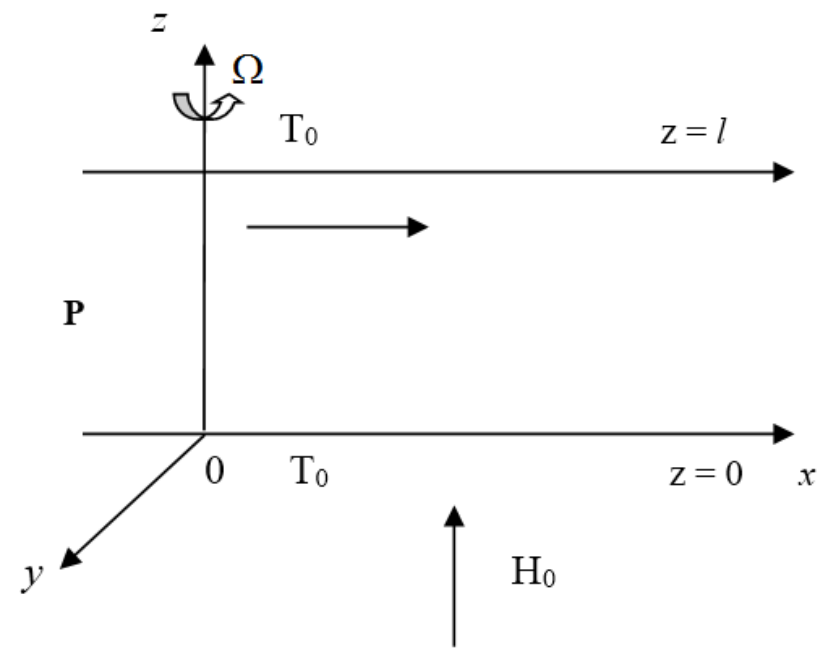

Figure 1. Physical configuration of the Problem

Using the non-dimensionalization process, the unsteady governing equations reduces to (dropping asterisks),

$$
\begin{aligned}
& \frac{\partial^{2} q}{\partial z^{2}}+S \frac{\partial^{3} q}{\partial z^{2} \partial t}-\left(M^{2}+2 i E^{-1}+D^{-2}\right) q-\frac{\partial q}{\partial t}=G r z H(t)+R \\
& \frac{\partial^{2} \theta}{\partial z^{2}}-\alpha \theta-\operatorname{Pr}\left(\frac{\partial \theta}{\partial t}+\left(G r_{1} u+G r_{2} v\right) H(t)\right)=0
\end{aligned}
$$

Where,

$M^{2}=\frac{\sigma \mu_{e}^{2} H_{0}^{2} l^{2}}{\rho v}$ is the Hartmann number (Magnetic field parameter),

$E=\frac{v}{\Omega l^{2}}$ is the Ekman number,

$S=\frac{\alpha_{1}}{\rho l^{2}}$ is the visco-elastic fluid parameter,

$D^{-2}=\frac{l^{2}}{k}$ is the inverse Darcy Parameter,

$P_{r}=\frac{\mu C_{p}}{k_{1}}$ is the Prandtl number,

$\alpha=\frac{Q l^{2}}{k_{1}}$ is the Heat source Parameter,

$R=\left(-\frac{l^{3}}{v^{3}}\right) D$ is the Pressure gradient Parameter,

$G r=G r_{1}+i G r_{2}$ is the Grashof number,
The corresponding initial and boundary conditions are

$$
\begin{aligned}
& q(z, t)=a e^{i \omega t}+b e^{-i \omega t} \text { at } z=0 \\
& q(z, t)=0 \text { at } z=1 \quad \forall t \leq 0, \forall z \\
& \theta(z, t)=0 \text { at } z=0 \forall t \leq 0, \forall z \\
& \theta(z, t)=\frac{\beta g l^{3}\left(\theta_{1} \omega_{2}-\theta_{1} \omega_{1}\right)}{v^{2}}=\theta_{0} \text { at } z=1
\end{aligned}
$$

Where all the physical quantities are used in this their usual meaning.

Making use of Laplace transforms technique, we solved the governing equations with relevant boundary conditions, we obtain the velocity and temperature.

The shear stress and Nusselt number at both plates are evaluated using the following,

$$
\begin{aligned}
& \rho_{L}=\left(\frac{d q}{d z}\right)_{z=0} \text { and } \tau_{v}=\left(\frac{d q}{d z}\right)_{z=1} \\
& (N u)_{L}=\left(\frac{d \theta}{d z}\right)_{z=0} \text { and }(N u)_{v}=\left(\frac{d \theta}{d z}\right)_{z=1}
\end{aligned}
$$

\section{RESULTS AND DISCUSSIONS:}

The analytical solution consists of the both steady and transient states. The quasi-steady parts of the velocity and temperature representing the ultimate flow have been computed numerically for different sets of governing parameters namely viz. The Hartmann parameter $M$, the inverse Darcy parameter $\mathrm{D}^{-1}$, the Ekman number $\mathrm{E}, \mathrm{S}$ second grade fluid parameter, Grashof number $\mathrm{Gr}$, the frequency of oscillation $\omega$, Prandtl number Pr and Heat source parameter $\alpha$, and their profiles are plotted in Figures (2-5) for the oscillating lower plate and for plates are in rest respectively. Figures (2-4) correspond to profiles for the velocity components $u$ and $v$, Figures (5) correspond to profiles for temperature when one of the plates (lower) is oscillating with given amplitude and other is at rest. Tables (1) represent the shear stresses at the stationary plate while table (2) signify the rate of heat transfer at both the plates.

It is evident from the figures (2-5) that the velocity profiles are parabolic in nature. We noticed that, the magnitudes of the velocity components $u$ enhance and $v$ diminish throughout the fluid region with increasing Ekman number E or Second grade fluid parameter $S$ being the parameters fixed (Fig $2 \& 5$ ). The resultant velocity is also increases with increasing $\mathrm{E}$ and $\mathrm{S}$. Both the velocity components $u$ and $v$ experiences retardation with increasing the intensity of the magnetic field (Hartmann number M). The application of the transverse magnetic field plays the important role of a resistive type force (Lorentz force) similar to drag force (that acts in the opposite direction of the fluid motion) which tends to resist the flow thereby decreasing its velocity (Fig 3). Similar behaviour is observed 
International Journal of Applied Engineering Research ISSN 0973-4562 Volume 13, Number 10 (2018) pp. 8200-8204

(C) Research India Publications. http://www.ripublication.com

for the resultant velocity. We observe that lower the permeability of the porous medium lesser the fluid speed in

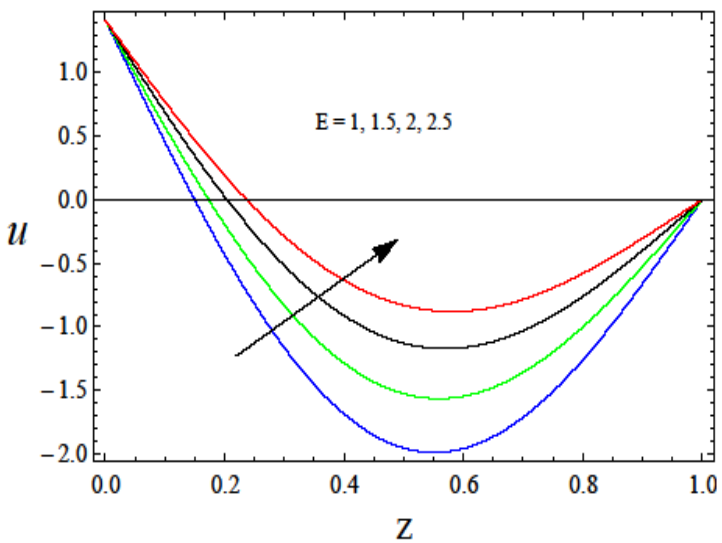

the entire fluid region. The resultant velocity is also trim downs throughout the fluid region.

Figure 2. The velocity profiles for $u$ and $v$ against $\mathrm{E}$

$$
M=1, D^{-1}=50, S=1, \omega=\pi / 4, G r=2
$$
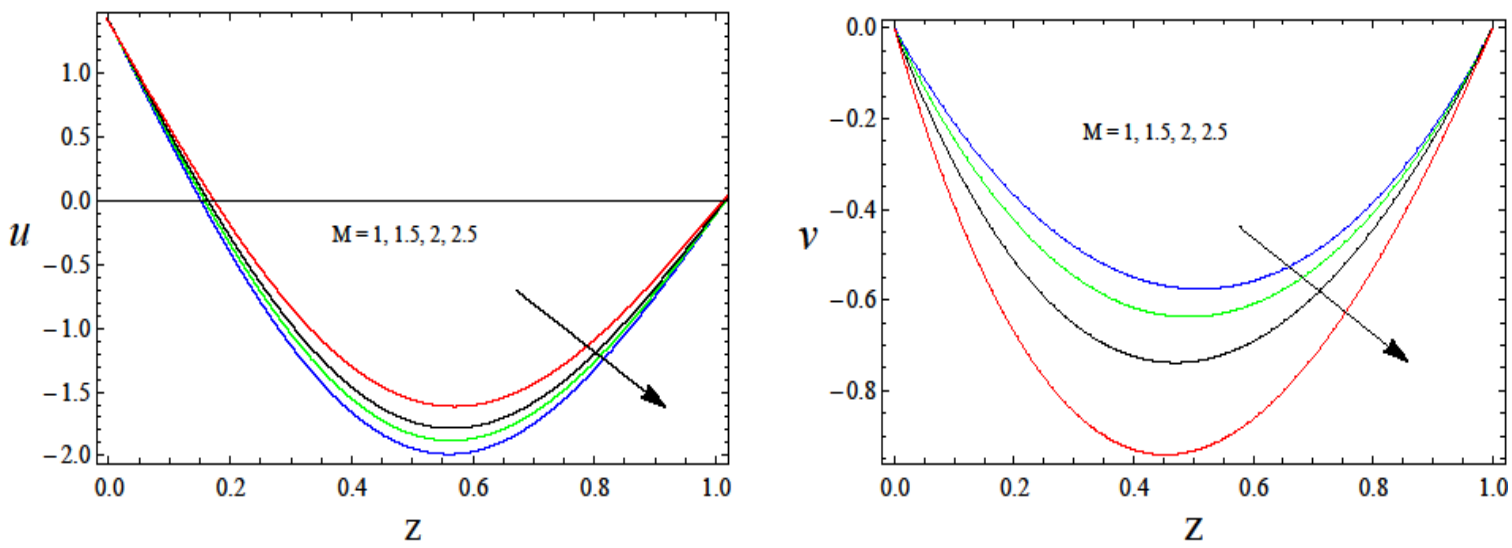

Figure 3. The velocity profiles for $u$ and $v$ against $\mathrm{M}$

$$
E=1, D^{-1}=50, S=1, \omega=\pi / 4, G r=2
$$
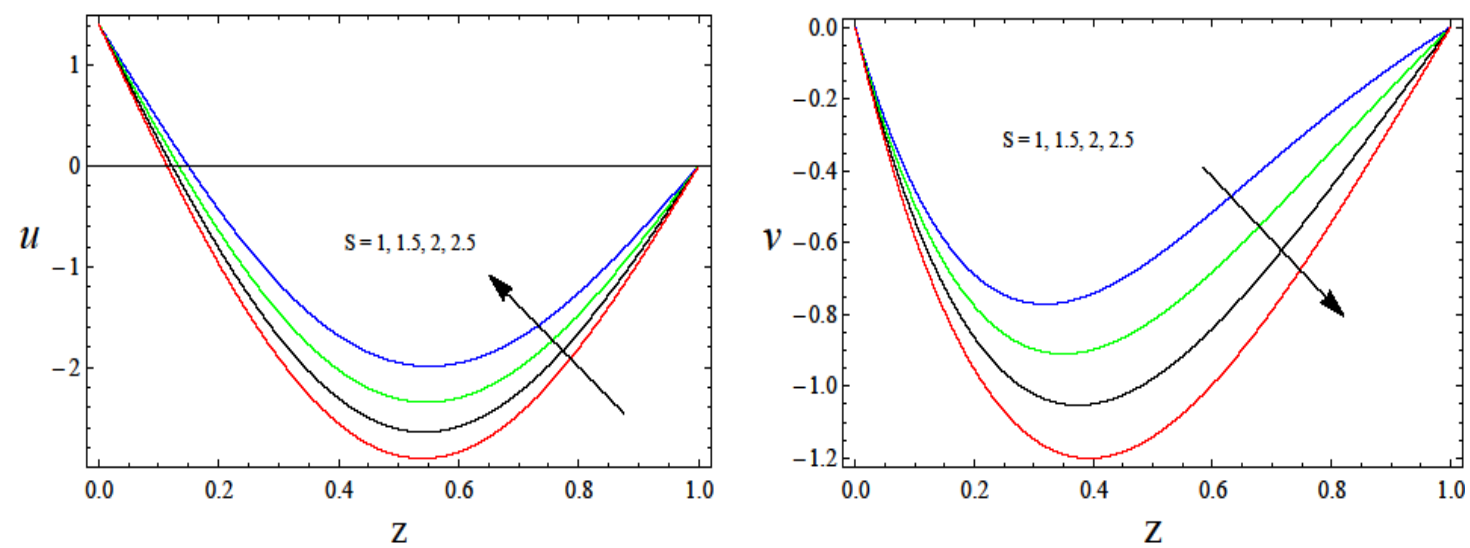

Figure 4. The velocity profiles for $u$ and $v$ against $\mathrm{S}$

$$
E=1, D^{-1}=50, M=1, \omega=\pi / 4, G r=2
$$


It is evident from the Fig (5) displays that the fluid temperature increases with an increase in Hartmann number M, Second grade fluid parameter $\mathrm{S}$, the inverse Darcy parameter $\mathrm{D}^{-1}$. The temperature reduces with increasing Ekman number E, Heat source parameter $\alpha$ and Grashof number Gr.

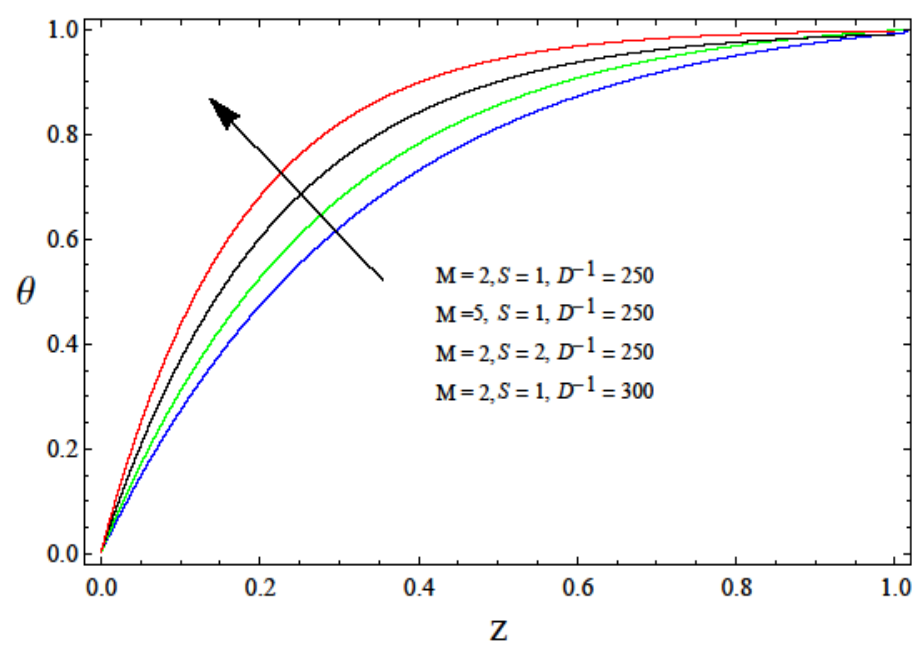

Figure 5. The Temperature Profiles for $\theta$ with $\mathrm{M}, \mathrm{S}$, and $\mathrm{D}^{-1}$

$$
E=1, G r=2, \alpha=5, \operatorname{Pr}=0.71, \omega=\pi / 4,
$$

The shear stresses $\left(\tau_{x} \& \tau_{y}\right)$ and Nusselt number $(\mathrm{Nu})$ are calculated computationally at the stationary plate and tabulated on the table (1). At the stationary plate, the magnitude of the stresses $\tau_{x}$ and $\tau_{y}$ enhances with increasing Ekman number $\mathrm{E}$ and the frequency of oscillation $\omega$. The reversal behavior is observed for increasing Grashof number Gr. The stresses $\tau_{x}$ reduce and $\tau_{y}$ increase in magnitude with increasing the intensity of the magnetic field $M$ or inverse Darcy parameter $\mathrm{D}^{-1}$, whereas stresses $\tau_{x}$ increases and $\tau_{y}$ decreases with increasing second grade fluid parameter $S$ (Table 1).

The magnitudes of the rate heat transfer $\mathrm{Nu}$ enhance with increasing Ekman number E, inverse Darcy parameter $\mathrm{D}^{-1}$, Grashof number Gr, the frequency of oscillation $\omega$ and Prandtl number Pr at stationary plate. The Nusselt number increases at stationary plate with increasing second grade fluid parameter $\mathrm{S}$, where as reversal trend observed with increasing the intensity of the magnetic field $\mathrm{M}$ or Heat source parameter $\alpha$ (Table 2).
Table 1: The Shear stress $\left(\tau_{x} \& \tau_{y}\right)$ on the stationary plate (upper plate)

\begin{tabular}{|c|c|c|c|c|c|c|c|}
\hline $\mathrm{E}$ & $\mathrm{M}$ & $\mathrm{D}^{-1}$ & $S$ & $\mathrm{Gr}$ & $\omega$ & $\tau_{x}$ & $\tau_{y}$ \\
\hline 1 & 2 & 100 & 1 & 5 & $\omega=\pi / 4$ & 2.524785 & -4.366588 \\
\hline 1.5 & 2 & 100 & 1 & 5 & $\omega=\pi / 4$ & 2.635525 & -4.988558 \\
\hline 2 & 2 & 100 & 1 & 5 & $\omega=\pi / 4$ & 2.855474 & -5.458851 \\
\hline 1 & 3 & 100 & 1 & 5 & $\omega=\pi / 4$ & 2.422458 & -8.457847 \\
\hline 1 & 5 & 100 & 1 & 5 & $\omega=\pi / 4$ & 2.002544 & -10.58854 \\
\hline 1 & 2 & 200 & 1 & 5 & $\omega=\pi / 4$ & 2.144581 & -5.478547 \\
\hline 1 & 2 & 300 & 1 & 5 & $\omega=\pi / 4$ & 1.520248 & -7.458874 \\
\hline 1 & 2 & 100 & 2 & 5 & $\omega=\pi / 4$ & 2.855255 & -3.221452 \\
\hline 1 & 2 & 100 & 3 & 5 & $\omega=\pi / 4$ & 3.554855 & -2.744666 \\
\hline 1 & 2 & 100 & 1 & 5 & $\omega=\pi / 4$ & 4.255857 & -5.141147 \\
\hline 1 & 2 & 100 & 1 & 5 & $\omega=\pi / 4$ & 6.477845 & -6.574855 \\
\hline 1 & 2 & 100 & 1 & 10 & $\omega=\pi / 4$ & 1.545587 & -2.448122 \\
\hline 1 & 2 & 100 & 1 & 15 & $\omega=\pi / 4$ & 0.378547 & -1.855479 \\
\hline 1 & 2 & 100 & 1 & 5 & $\omega=\pi / 3$ & 6.665485 & -7.145799 \\
\hline 1 & 2 & 100 & 1 & 5 & $\omega=\pi / 2$ & 8.788514 & -9.558546 \\
\hline
\end{tabular}

Table 2: The Rate of Heat transfer (Nusselt number) at stationary plate

\begin{tabular}{|c|c|c|c|c|c|c|c|c|c|}
\hline $\mathrm{E}$ & $\mathrm{M}$ & $\mathrm{D}^{-1}$ & $S$ & $m$ & $\mathrm{Gr}$ & $\omega$ & $\alpha$ & $\mathrm{Pr}$ & Stationary plate \\
\hline 1 & 2 & 100 & 1 & 1 & 5 & $\omega=\pi / 4$ & 5 & 0.71 & 1.588748 \\
\hline 1.5 & 2 & 100 & 1 & 1 & 5 & $\omega=\pi / 4$ & 5 & 0.71 & 2.554887 \\
\hline 2 & 2 & 100 & 1 & 1 & 5 & $\omega=\pi / 4$ & 5 & 0.71 & 3.221142 \\
\hline 1 & 3 & 100 & 1 & 1 & 5 & $\omega=\pi / 4$ & 5 & 0.71 & 1.255447 \\
\hline 1 & 5 & 100 & 1 & 1 & 5 & $\omega=\pi / 4$ & 5 & 0.71 & 0.855475 \\
\hline 1 & 2 & 200 & 1 & 1 & 5 & $\omega=\pi / 4$ & 5 & 0.71 & 2.855478 \\
\hline 1 & 2 & 300 & 1 & 1 & 5 & $\omega=\pi / 4$ & 5 & 0.71 & 3.988758 \\
\hline 1 & 2 & 100 & 2 & 1 & 5 & $\omega=\pi / 4$ & 5 & 0.71 & 1.885574 \\
\hline 1 & 2 & 100 & 3 & 1 & 5 & $\omega=\pi / 4$ & 5 & 0.71 & 2.155245 \\
\hline 1 & 2 & 100 & 1 & 2 & 5 & $\omega=\pi / 4$ & 5 & 0.71 & 5.788547 \\
\hline 1 & 2 & 100 & 1 & 3 & 5 & $\omega=\pi / 4$ & 5 & 0.71 & 8.966585 \\
\hline 1 & 2 & 100 & 1 & 1 & 10 & $\omega=\pi / 4$ & 5 & 0.71 & 5.785547 \\
\hline 1 & 2 & 100 & 1 & 1 & 15 & $\omega=\pi / 4$ & 5 & 0.71 & 8.477485 \\
\hline 1 & 2 & 100 & 1 & 1 & 5 & $\omega=\pi / 3$ & 5 & 0.71 & 1.988548 \\
\hline 1 & 2 & 100 & 1 & 1 & 5 & $\omega=\pi / 2$ & 5 & 0.71 & 2.688995 \\
\hline 1 & 2 & 100 & 1 & 1 & 5 & $\omega=\pi / 4$ & 8 & 0.71 & 1.255485 \\
\hline 1 & 2 & 100 & 1 & 1 & 5 & $\omega=\pi / 4$ & 10 & 0.71 & 0.007588 \\
\hline 1 & 2 & 100 & 1 & 1 & 5 & $\omega=\pi / 4$ & 5 & 7 & 2.855479 \\
\hline
\end{tabular}




\section{CONCLUSIONS}

1. The resultant velocity is increases with increasing $\mathrm{E}$ or $\mathrm{S}$.

2. An increase in Grashof number leads to raise both the primary velocity $u$ and the secondary velocity $v$.

3. The fluid temperature increases with an increase in $\mathrm{M}, \mathrm{S}, \mathrm{D}^{-1}, \operatorname{Pr}$ and $\omega$.

4. The magnitude of the both stresses enhances with increasing $\mathrm{E}$ and $\omega$, also reduces for increasing $\mathrm{Gr}$ at the stationary plate.

5. The magnitude of the both stresses enhances with increasing $\mathrm{E}, \mathrm{D}^{-1}$ and $\mathrm{S}$ at the oscillatory plate. The stresses reduce in magnitude with increasing $\mathrm{M}, \mathrm{Gr}$ or $\omega$.

6. The magnitudes of the rate heat transfer $(\mathrm{Nu})$ enhance with increasing $\mathrm{E}, \mathrm{D}^{-1}, \mathrm{Gr}, \omega$ and $\operatorname{Pr}$ at either plates.

\section{Conflict of interest:}

The authors are not interested the conflict of interest on this paper

\section{REFERENCES}

[1]. Singh, K.D. and Alphonsa, M. (2008), "Injection/Suction Effects on an Oscillatory Hydromagnetic Flow in a Rotating Horizontal Porous Channel," Indian Journal of Physics, 82, 435-445.

[2]. Veera Krishna, M. and Suneetha, S.V. (2009), "Hall Effects on Unsteady MHD Rotating Flow of an Incompressible Viscous Fluid through a Porous Medium," Journal of Pure and Applied Physics, 21, 143-156.

[3]. Das, S., Mandal, H.K. and Jana, R.N. (2013), "Hall Effect on Unsteady MHD Flow through a Porous Channel in a Rotating System with Variable Pressure Gradient," Ph.D. Thesis, Vidya Sagar University, Midnapur, 1-23.

[4]. M. Narahari (2010), "Effects of thermal radiation and free convection currents on the unsteady Couette flow between two vertical parallel plates with constant heat flux at one boundary," WSEAS Transactions on Heat and Mass Transfer, 5(1), 21-30.

[5]. S. S. Das, S. R. Biswal, U. K. Tripathy, and P. Das (2011), "Mass transfer effects on unsteady hydromagnetic convective flow past a vertical porous plate in a porous medium with heat source," Journal of Applied Fluid Mechanics, 4(4), 91-100.

[6]. I. Khan, K. Fakhar, and S. Shafie (2011), "Magnetohydrodynamic free convection flow past an oscillating plate embedded in a porous medium," Journal of the Physical Society of Japan, 80(10), Article ID 104401.
[7]. A. Khan, I. Khan, F. Ali, S. Ulhaq, and S. Shafie (2014), "Effects of wall shear stress on unsteady MHD conjugate flow in a porous medium with ramped wall temperature," PLoS ONE, 9(3), Article ID e90280.

[8]. A. Hussanan, Z. Ismail, I. Khan, A. G. Hussein, and S. Shafie (2014), "Unsteady boundary layer MHD free convection flow in a porous medium with constant mass diffusion and Newtonian heating," The European Physical Journal Plus, 129, 46.

[9]. R. N. Barik, G. C. Dash, and M. Kar (2013), "Free convection heat and mass transfer MHD flow in a vertical porous channel in the presence of chemical reaction," Journal of Fluids, , Article ID 297493, 14 pages, 2013.

[10]. M.Veera Krishna and J.Prakash (2015), "Hall current effects on Unsteady MHD flow in a Rotating parallel plate channel bounded by Porous bed on the Lower half - Darcy Lapwood model," Open Journal of Fluid Dynamics, 5, 275-294. http://dx.doi.org/10.4236/ojfd.2015.54029.

[11]. M.VeeraKrishna, "Unsteady MHD Flow in a Rotating Parallel Plate Channel under the Influence of Pressure Gradient with Hall Current Effects," Journal of Scientific Research and Reports, 10(3), 1-21. DOI: 10.9734/JSRR/2016/24123.

[12]. B.Lakshmanna and S.Venkateswarlu (2017), “Journal of Ultra scientist of physical sciences”(JUSPB),Vol.29(9),234-247.

[13]. B.Lakshmanna and S.Venkateswarlu (2018), "Hall effects on MHD flow of second grade fluid through porous medium with oscillatory suction", International journal of applied engineering research, Vol.13 (7), pp. 4782-4790.

[14]. B.Lakshmanna and S.Venkateswarlu (2018), "Unsteady MHD Convective Flow of an Incompressible viscous fluid through porous medium", Annals of pure and applied mathematics, Vol.16 (2), pp.451-460. 